\title{
Miranda
}

Revue pluridisciplinaire du monde anglophone /

Multidisciplinary peer-reviewed journal on the English-

speaking world

$12 \mid 2016$

Mapping gender. Old images ; new figures

This is what feminisms look like

Lutter contre les violences sexuelles, (re)présenter

un sujet politique

\section{Pauline Delage}

\section{OpenEdition}

Journals

Édition électronique

URL : http://journals.openedition.org/miranda/8618

DOI : 10.4000/miranda.8618

ISSN : 2108-6559

\section{Éditeur}

Université Toulouse - Jean Jaurès

\section{Référence électronique}

Pauline Delage, "This is what feminisms look like

Lutter contre les violences sexuelles, (re)présenter un sujet politique », Miranda [En ligne], 12 | 2016,

mis en ligne le 02 mars 2016, consulté le 16 février 2021. URL : http://journals.openedition.org/

miranda/8618 ; DOI : https://doi.org/10.4000/miranda.8618

Ce document a été généré automatiquement le 16 février 2021.

\section{cc)}

Miranda is licensed under a Creative Commons Attribution-NonCommercial-NoDerivatives 4.0

International License. 


\title{
This is what feminisms look like Lutter contre les violences sexuelles, (re)présenter un sujet politique
}

\author{
Pauline Delage
}

Pendant trois jours, des dizaines de tee-shirts de couleurs différentes sont suspendus à des fils à

linge dans l'une des cours de l'University of California Los Angeles. Sur ces tee-shirts, des textes et des dessins sont inscrits pour dénoncer ou témoigner des violences sexuelles et conjugales. Intrigué-e-s, des étudiant-e-s de l'université s'arrêtent pour regarder et lire les messages. A côté de cet accrochage, un espace à l'abri des regards est réservé à celles et ceux qui souhaitent s'approprier un tee-shirt pour y écrire un témoignage sur des violences. Chaque année, cette action, The Clotheline Project ${ }^{1}$, a lieu pour « rompre le silence » sur les violences et en « parler».

Carnet de terrain, UCLA, mai 2011.

1 Au début des années 1980, le féminisme a parfois pu être envisagé comme un mouvement «mort, fini, kaput, nada $»^{2}$. A l'encontre d'un tel discours, des auteures ont préféré souligner les reconfigurations des mobilisations, en montrant que l'idée de leur déclin découle d'une définition restrictive du féminisme ou encore du " point de vue situé $\|^{3}$ de leurs observatrices, militantes et chercheuses. En effet, si les années 1980 correspondent à une période de backlash, elles renvoient également à un redéploiement des actions politiques au sein des mondes associatifs et institutionnels ${ }^{4}$ : des centres d'accueil pour femmes victimes de violences sexuelles et conjugales se multiplient, des 
actrices ouvrent des cliniques pour proposer des avortements, et d'autres se concentrent sur la réforme des politiques publiques. C'est aussi le moment où des femmes issues des minorités sexuelles et raciales remettent en cause l'hégémonie des revendications formulées par des femmes majoritairement blanches, hétérosexuelles et de classe moyenne ${ }^{5}$. Aussi le féminisme est-il un mouvement pluriel, difficilement délimité et délimitable, tant du point de vue politique que sociologique ${ }^{6}$. Autrement dit, les mouvements féministes ne se sont pas éteints, mais leur objet et le sujet politique qu'ils sont censés représenter ont évolué et sont controversés. Prendre l'exemple de la lutte contre les violences sexuelles ${ }^{7}$ permet de se départir d'une analyse en termes de stricte dépolitisation pour mieux comprendre les formes d'appropriation des motifs et des modes d'action élaborés dans les années 1970, ainsi que les transformations dans les politiques de représentation féministe.

2 En confrontant l'histoire de la dénonciation des violences, qui apparaît au début des années 1970, à des modes d'action plus récents, émergeant dans les années 1990, comme The Clothesline Project, on peut en effet mettre en lumière certaines des continuités et des ruptures entre des contextes de mobilisation et des espaces féministes variés. The Clothesline Project est donc ici envisagé comme l'un des cas illustrant les reconfigurations des féminismes et l'évolution de la question de la représentation politique, c'est-à-dire des formes et du sujet de la représentation ${ }^{8}$. Pour ce faire, cet article se fonde sur une partie d'une enquête ethnographique menée principalement dans des associations de lutte contre la violence conjugale dans le comté de Los Angeles et qui a donné lieu à un travail d'observation à University of California Los Angeles ${ }^{9}$. Trois temps, et trois questions, le rythmeront. Premièrement, comment la question du viol et des violences sexuelles a-t-elle été problématisée par les militantes féministes ? Deuxièmement, en quoi The Clothesline Project constitue-t-il une forme d'appropriation ${ }^{10}$, c'est-à-dire à la fois un prolongement et une transformation, du répertoire d'action développé par les féministes des années 1970 ? Pour finir, quel type de sujet politique est représenté à travers cette action? On verra alors la manière dont l'action se structure autour de l'acte de violence et met en avant la figure de le/la survivant-e, aux dépens d'un sujet politique plus globalisant, comme celui des «femmes».

\section{Dénoncer le viol, aider les victimes}

Bien que les violences sexuelles aient toujours existé, elles n'ont pas nécessairement été considérées comme un problème en tant que tel ${ }^{11}$. La question du viol est en effet peu prise en compte dans les politiques publiques aux États-Unis jusque dans les années 1970 à l'époque où les militantes féministes soulignent l'universalité du phénomène et son ancrage dans des rapports sociaux de sexe. Parfois politisées au sein de la gauche ou du mouvement de libération des femmes, dans les groupes de conscience par exemple, certaines actrices s'emploient à dévoiler ce problème en rompant avec le silence social qui l'entoure ${ }^{12}$. Se multiplient alors à la fin des années 1960 et au début des années 1970 les textes qui soulignent l'ampleur et la fonction sociale du viol ainsi que les actions de dénonciation. 


\section{Le viol comme problème}

Publié en 1971, «Rape : The All-American Crime $»^{13}$ est l'un des tous premiers textes qui porte spécifiquement sur le viol et ses rouages sociaux. A rebours d'une vision naturalisante, Susan Griffin, auteure de l'article, montre à quel point le viol est central dans l'histoire culturelle états-unienne, ce qui contribue en retour à le rendre légitime. C'est bien la socialisation sexuée qui façonne la prétendue vulnérabilité des femmes et le rapport des hommes à la sexualité et au pouvoir, la naturalisation des rôles sexués renforçant la prégnance des violences sexuelles dans la société. Autre texte fondateur de la lutte contre la violence, le livre de Susan Brownmiller, Against our Will ${ }^{14}$, étudie également les mécanismes historiques, sociaux et religieux qui rendent le viol possible et légitime dans le monde social. Plus que des attaques isolées, les violences sexuelles et leur menace, c'est-à-dire leur potentialité, fonctionnent comme des vecteurs de contrôle du corps des femmes: craignant de subir des violences, les femmes sont incitées à rester au foyer, dans la sphère privée. Nombre de publications dans des revues ou des lettres d'information ${ }^{15}$ vont alimenter la compréhension du viol en tant que produit de la domination masculine. Ainsi, les violences sexuelles ne sont plus comprises comme un épiphénomène, causé par quelque déviance individuelle, d'ordre pathologique ou social, elles deviennent un problème en soi, qui est généré par des rapports de domination et qui renforce ces derniers.

\section{Des femmes aux femmes victimes}

Pour lutter contre l'invisibilité des violences, les féministes se mobilisent dans l'espace public en organisant notamment des actions de rue, des manifestations, comme Take Back the Night dont l'objectif est de permettre aux femmes de se réapproprier collectivement la rue ${ }^{16}$, ainsi que des speak outs qui sont des rassemblements où les femmes témoignent de leur expérience du viol pour rappeler que toutes en sont victimes, directement ou indirectement. Décrit dans l'ouvrage de Susan Brownmiller, le premier speak-out est organisé par les New York Radical Feminists en 1971. Pour l'auteure, c'est l'un des premiers moments où la prégnance des violences sexuelles l'interpelle ; elle prend alors conscience du poids qu'a la menace de viol dans la subjectivation des femmes. Si elle avait jusqu'alors montré une certaine distance à l'égard de la politisation des violences, elle se rend compte qu'elle fait bien partie de ce collectif, non pas en tant que victime, mais en tant que femme. Considéré comme le révélateur et le symptôme de l'oppression structurelle que toutes les femmes subissent, le viol fait partie de leur expérience commune ; il façonne nécessairement leur rapport au corps, à l'espace public et à la mobilité. Ainsi, ce sont principalement les femmes, plutôt que les victimes, qui sont le sujet central de ces mobilisations. S'agissant des revendications féministes généralistes et celles spécifiques au viol, la centralité du sujet "femmes» dans les revendications a toutefois fait l'objet de polémiques. L'idée d'un «nous les femmes » a en effet été contestée par les féministes noires et latinas qui ont montré que ce collectif fondé sur une même identité sexuée occultait les privilèges socio-raciaux de celles qui s'en réclament. De la même manière, Angela Davis a mis en relief les biais racistes des travaux de Susan Brownmiller, plus encline, selon la militante féministe noire, à dénoncer les viols commis par des hommes noirs ${ }^{17}$. La judiciarisation et la multiplication des dispositifs d'aide aux victimes vont cependant opérer un glissement 
dans la construction du sujet des mobilisations contre les violences, passant des femmes aux femmes victimes.

6 Conjointement à l'analyse du phénomène social, les militantes proposent une critique des différentes institutions qui participent de la négation du viol, en responsabilisant les femmes victimes et, parallèlement, en faisant preuve d'une certaine complaisance à l'égard des agresseurs. La justice et le droit sont l'une des principales cibles. Bien qu'il soit fréquent, le viol est en effet rarement pris en charge par les forces de l'ordre et condamné dans les tribunaux. Tout en manifestant une certaine défiance à l'égard des institutions juridiques, certains groupes se sont donc attachés à dénoncer publiquement un droit inégalitaire et à agir pour le réformer ${ }^{18}$. Les services de police ont par exemple été incités à ne plus adopter une attitude culpabilisante et à recueillir systématiquement les plaintes des femmes. A travers la réforme du code pénal des différents États de l'Union, la définition du viol a aussi changé pour désigner tout rapport sexuel non-consenti dans le cadre de relations intimes, familiales et conjugales ou des violences perpétuées par un inconnu ${ }^{19}$. Ainsi, en 1977, l'Oregon est le premier État à annuler une clause d'exemption maritale permettant la qualification pénale du viol conjugal. Ce type de réforme se généralise par la suite. L'approche féministe juridique consiste tout autant en une lutte pour la reconnaissance d'un problème public que pour celle du statut de victime. Liée au mouvement de spécialisation croissante de groupes militants, la focalisation progressive sur les victimes fait écho à la mise en place des rape crisis centers (centres anti-viol) ${ }^{20}$, qui se développent à partir de 1972 dans plusieurs villes, à Washington D.C., Berkeley, Detroit ou encore Philadelphie. En 1976, il $\mathrm{y}$ aurait environ quatre-cents rape crisis centers ${ }^{21}$.

7 Souvent structurés autour d'une ligne d'écoute, les rape crisis centers sont des lieux où les femmes peuvent dire les violences vécues et bénéficier d'un accompagnement social et juridique. Divers d'un point de vue politique, tous ne sont pas issus de groupes féministes, certains étant plus ou moins rattachés à des agences de santé mentale. Cependant, la plupart sont des «produits " $^{22}$ des mouvements féministes, et ils proposent un travail politique de dénonciation des violences qui imprègne les pratiques d'aide et la relation de service. Il s'agit, en effet, pour les bénévoles et les salariées de ces centres de prendre en compte les besoins et les choix des femmes afin d'éviter toute forme de responsabilisation et de culpabilisation. Les principes politiques défendus par les rape crisis centers féministes se traduisent également par l'importance accordée à la promotion de réformes ainsi qu'à la prévention des violences, voire aux actions publiques de dénonciation. En déplaçant la focale de la lutte contre l'oppression des femmes vers celle contre les violences sexuelles, les militantes ont façonné un nouveau sujet, celui de "femmes victimes", qui s'est progressivement imposé dans la lutte contre les violences.

8 A l'extérieur des associations spécialisées dans le traitement des violences sexuelles, d'autres actions sont menées pour rendre visible ce problème. L'université, autre sphère dans laquelle les féminismes s'institutionnalisent, devient l'un des espaces où la question du viol est posée ${ }^{23}$.

\section{Appropriation et renouvellement des modes d'actions}

9 Apparu sur le campus de Cape Cod, Massachusetts, à partir de 1990, The Clothesline Project est une performance organisée annuellement sur certains campus des États- 
Unis $^{24}$. Cette action prend forme dans un contexte où les féminismes médiatiques et universitaires sont traversés par des polémiques autour de l'importance du date rape, c'est-à-dire du viol lors d'un rendez-vous amoureux, sur les campus états-uniens ${ }^{25}$. Relayé dans les revues féministes Ms. Magazine et Off Our Backs, The Clothesline Project a pris une ampleur nationale et a dorénavant lieu dans les universités de quarante-et-un Etats différents. Dans le jardin de UCLA par exemple, les participant-e-s $\mathrm{s}^{26}$ exposent des tee-shirts de couleurs différentes symbolisant des survivors (survivantes) de violences sexuelles. Sans nécessairement présenter la question des violences sexuelles de la même manière sur chaque campus, The Clothesline Project est une stratégie d'action qui se revendique féministe et qui vise à une appropriation de l'espace public, en mettant en avant la parole des personnes concernées. En ce sens, il s'ancre dans l'histoire d'un répertoire d'action féministe, c'est-à-dire "un modèle où l'expérience accumulée d'acteurs s'entrecroise avec les stratégies d'autorités, en rendant un ensemble de moyens d'action limités plus pratique, plus attractif, et plus fréquent que beaucoup d'autres moyens qui pourraient, en principe, servir les mêmes intérêts $»^{27}$. Tout en rompant avec certains principes politiques féministes, Clothesline prolonge les modes d'action développés dans les années 1970 ; il révèle ainsi les formes d'appropriation du répertoire d'action militant et de la manière de comprendre et d'expliquer le phénomène des violences sexuelles.

\section{L'héritage radical} différenciation des sphères privée et publique, The Clothesline Project se fonde sur des schèmes de pensée proches de ceux qu'illustrent les speak-outs et Take Back the Night, principalement initiés par des groupes féministes radicaux dans les années 1970. Il est, là encore, question de décloisonner les sphères en introduisant dans l'espace public un problème relégué aux domaines du privé et de l'intime. Dévoiler ce problème et le politiser à travers une forme d'expression collective prolongent alors un bouleversement des frontières, entre ce qui est de l'ordre du privé, du public et du politique.

Tout comme les manifestations de nuit, qui sont envisagées comme un moyen pour les femmes de s'emparer de la sphère publique, The Clothesline Project est une action publique qui se déroule à l'extérieur dans un lieu de passage - un jardin ou une cour pour rendre le phénomène visible et créer des espaces d'expression que les survivors peuvent s'approprier. Rappelons d'ailleurs que les manifestations ou les événements se réclamant de la démarche de Take Back the Night existent toujours aux États-Unis ${ }^{28}$. L'héritage féministe est ici relativement explicite dans la mesure où les fondatrices de Clothesline revendiquent le fait d'avoir participé à des manifestations comme Take Back the Night au moment de l'élaboration de ce projet.

En outre, The Clothesline Project s'inscrit dans la tradition des speak-outs dont l'objectif premier est de faire émerger la parole des femmes, de rompre le silence social sur les violences sexuelles en participant de la subjectivation des femmes victimes. Si la question de la prise de parole est centrale, ce n'est pas uniquement à cause du caractère spécifique des violences sexuelles, de leur manque de reconnaissance sociale et juridique, mais plus généralement parce que la parole est pensée comme un outil d'autonomisation des femmes. Façonnée par le genre, la prise de parole est répartie et 
rendue légitime de façon asymétrique dans le monde social, celle émanant d'hommes étant généralement mieux considérée et davantage entendue que celles des femmes. Les féministes considèrent donc que s'affirmer en parlant, entre femmes notamment, contribue à l'émancipation de chacune. Ainsi la question de la parole est-elle un levier vers la constitution d'un sujet collectif. Régie par des fondements politiques et cognitifs semblables à ceux des groupes de conscience (consciousness-raising groups), l'expression individuelle du viol doit contribuer à créer une identité collective, sexuée, fondée sur l'expérience d'une oppression commune. De la même façon, l'idée fondamentale de Clothesline est de créer un espace pour nommer les violences.

S'il se fonde sur une importance accrue accordée à l'expression et au témoignage, The Clothesline Project demeure différent des speak-outs et des groupes de conscience. Les tee-shirts ne sont pas seulement les symboles d'un problème social qu'il convient de rendre visible; ils constituent de véritables supports pour la parole des survivors, ces derniers-ières étant encouragé-e-s à rédiger quelques mots sur ces tee-shirts pour témoigner : "Chacun des tee-shirts porte le message de quelqu'un qui a survécu à un viol, à des violences, à de l'inceste ou à des crimes de haine. Bien que quelques teeshirts soient faits en souvenir de ceux et celles qui n'ont pas survécu, certains de ces messages montrent de la colère; certains sont vulgaires, d'autres sont plus indulgents et d'autres encore exposent des faits. The Line permet aux survivant-e-s qui y participent de s'exprimer sans crainte ${ }^{29}$. En incarnant les victimes, ces tee-shirts sont un medium entre elles et le reste du monde social. Sans toujours être revendicatifs, les messages contribuent toutefois à publiciser la question des violences en mobilisant la figure $\mathrm{du} / \mathrm{de}$ la survivor et celle du témoin.

La forme de l'action révèle la volonté, sinon de subvertir, du moins d'interroger les délimitations entre les espaces sociaux. Le nom de l'événement, le fil à linge, renvoie aussi au travail ménager et symbolise ainsi une part invisible et gratuite des activités auxquelles les femmes sont assignées. Le fait de le rendre visible traduit encore une fois le lien entre la division sexuée des espaces et les violences.

\section{Transformations des modes d'action}

Héritier des mobilisations féministes radicales à certains égards, The Clothesline Project revèle pourtant des transformations importantes dans le répertoire d'action militant. Le type d'action, proche de la performance artistique, le lieu où elle se déroule ainsi que sa temporalité, qu'on qualifie d'événementielle, reflètent des évolutions dans les modes d'organisation et des objectifs de l'action collective. Ponctuelle et ciblée, cette action politique s'appuie sur un support artistique. Plusieurs facteurs permettent d'expliquer pourquoi ce type de médium a pu être utilisé : les transformations des répertoires d'action à partir des années 1980 et le fait que le registre culturel et artistique a été particulièrement investi d'une part, le profil sociologique des participantes à The Clothesline Project d'autre part. Tout d'abord, bien que la question des contours d'un féminisme culturel ${ }^{30}$, principalement orienté vers la promotion d'une culture féminine, lesbienne et féministe ait pu être discutée, les groupes de musique, dont l'emblématique Riot Grrrls ${ }^{31}$ est probablement le plus connu, et les expositions artistiques se réclamant des féminismes se sont multipliés à partir des années 1980 et 1990. Du point de vue sociologique ensuite, les personnes qui ont lancé cette action à Cape Cod, mais aussi plus tard à UCLA, sont étudiantes en arts plastiques, formation qui 
leur a permis d'acquérir un ensemble de dispositions à déployer dans l'organisation collective.

Par ailleurs, le lieu de l'action n'est pas non plus anodin puisqu'avec la politisation du date rape, l'université est progressivement repensée comme un espace où des violences sexistes s'exercent ${ }^{32}$. La politisation du problème des violences sexuelles à l'université se reflète dans les politiques publiques, avec notamment la mise en place du Campus Security Act en 1990, plus connu sous le nom de Jeanne Clery Act, à la suite du viol et du meurtre de Jeanne Clery. Ainsi, l'action The Clothesline project entre tout à fait en résonance avec le développement d'une critique féministe de type artistique et son déploiement à l'université. Le fait qu'il s'agisse d'un événement annuel peut également être envisagé comme l'une des évolutions des modes d'organisation collective. Le groupe que j'ai observé se réunit spécifiquement pour organiser cet événement, et ses membres ne sont d'ailleurs pas toujours engagés dans l'espace féministe. Certain-e-s des participant-e-s ont des liens forts avec d'autres associations féministes, mais c'est une association étudiante mixte qui s'est constituée spécifiquement et qui se réunit pour la semaine Clothesline : le calendrier politique est donc pré-déterminé, et l'identité féministe de ce groupe ne se constitue qu'au travers de l'événement. Dans la mesure où l'action et les modalités de l'action sont pré-établies, les réunions ne visent qu'à régler les problèmes pratiques: le nombre de tee-shirts à commander, le lieu de la performance, etc. Il y a donc un avant «pratique » de l'action, le moment de l'action, sans qu'il n'y ait d'après, sinon le renouvellement de The Clothesline. La spécificité organisationnelle participe à renforcer une dichotomie entre victimes et personnes engagées dans la lutte contre le viol. Non pas que les personnes engagées ne soient pas parfois victimes, mais l'action n'est pas l'occasion de la formation d'un collectif plus pérenne entre les victimes et les personnes engagées dans la lutte contre les violences. Les conditions de mise en forme de l'action façonnent le cadrage du problème des violences et le sujet politique mis en avant par The Clothesline project.

\section{Présenter un problème, construire un sujet}

Outre le fait de créer un espace d'expression, The Clothesline project est envisagé comme une manière de constater et de témoigner du problème des violences, plutôt que comme un mode de construction d'un sujet collectif. La notion de témoignage a pris une place grandissante dans la modernité tardive, et a été travaillée en sociologie, notamment à travers les survivants d'Auschwitz ${ }^{33}$ : qu'est-ce que témoigner ? Comment le faire? Pour quoi? Et pour qui? La réponse apportée ici est une forme de semivisibilité puisque l'action consiste à recueillir une parole, limitée par le format même de l'action, sans qu'elle soit susceptible de donner lieu à un mouvement collectif ${ }^{34}$. Il s'agit d'une certaine manière de rappeler à ceux et celles qui n'ont pas été victimes que les violences existent, et à celles qui l'ont été qu'elles ne sont pas seules. Par rapport à la perspective féministe des années 1970, deux types de glissements caractérisent le cadrage de The Clothesline Project : le premier est lié au problème traité - de la lutte contre la domination ou l'oppression des femmes aux violences sexuelles, celles-ci étant fragmentées en différentes sous-catégories -, le second touche au sujet politique. A travers cette action, la multiplicité du problème des violences sexuelles et la figure des survivors sont mises en avant. 


\section{Des violences sexuelles multiples}

Dans The Clothesline, la question des violences sexuelles apparaît comme un problème divisé en différentes catégories, pour lequel la victimation et le fait d'être un-e survivor sont le seul dénominateur commun. Les couleurs des tee-shirts exposés varient en fonction du type de violence vécue. Par exemple, le blanc représente les femmes mortes à cause des violences; le jaune ou le beige celles qui ont subi des violences conjugales (" battered or assaulted women ») ; le rouge, rose ou orange, les survivant-e-s de viols et de violences sexuelles ; le bleu et le vert, les survivant-e-s d'inceste et d'abus sexuels; le violet représente celles qui ont été agressées à cause de leur orientation sexuelle; le noir pour des raisons politiques ${ }^{35}$. Ainsi, si les violences sexuelles ont avant tout été politisées comme le produit des rapports de domination, cette cause commune tend à se dissiper dans la diversité des expériences et des cas de violence possibles.

La manière de cadrer le problème varie cependant en fonction des contextes locaux. Alors que l'objectif de l'action présenté à UCLA est de "faire prendre conscience des problèmes de violences sexuelles et des violences de genre sur le campus et dans le monde ${ }^{36}$, sur le site national, l'accent est mis sur les violences faites aux femmes, et il est davantage question " de faire mieux connaître l'impact des violences faites aux femmes, de célébrer la force d'une femme qui a survécu aux violences et de donner le courage de briser le silence qui entoure souvent son expérience ${ }^{37}$. Dans le cas de UCLA, les violences faites aux gays est ainsi prise en compte plus explicitement, mais les rapports sociaux au fondement des violences faites aux femmes sont éludés. Quelle que soit l'approche des violences privilégiée, dans la mesure où ces couleurs sont la première chose que remarquent les passant-e-s, cette mise en scène met en évidence les différentes catégories de violences. Ainsi, l'action ne met pas en relief un unique problème social ou un «ennemi principal $»^{38}$, mais bien différents problèmes. L'insistance sur la diversité des violences découle à la fois de la spécialisation des mouvements féministes et de la complexification du problème des violences sexuelles. Pourtant, le fait de genrer ou non les victimes reste le site de tensions qui soulignent une grande hétérogénéité des perspectives dans la lutte contre les violences. Alors que les violences faites aux femmes ont été les premières à concentrer l'attention des militantes, celles subies par les hommes, notamment gays ou bisexuels, et par les enfants bénéficient d'une reconnaissance croissante. Au problème original dénoncé par les féministes - l'oppression des femmes - se substitue celui de l'acte subi, la violence, segmenté en différents problèmes sociaux. De la même manière, à UCLA, le sujet de l'action n'est pas genré : on évoque les survivors (survivant-e-s), une formulation censée valoriser le potentiel agissant des survivantes.

\section{Des survivors}

Avec la notion de survivor, il s'agit de mettre en lumière la capacité d'agir des personnes, le fait que les victimes ne sont pas victimes à vie, et donc de se démarquer de toute forme de victimisation ${ }^{39}$. Bien entendu, un-e survivor devient survivant-e dès lors qu'elle a vécu des violences, que celles-ci aient lieu dans le cadre conjugal, familial ou non, qu'elles soient d'ordre sexuel ou non. C'est cependant l'empowerment des survivant-e-s qui est valorisé. S'il n'entre pas à proprement parler en rupture avec les féminismes des années 1970, le concept d'empowerment revêt un sens différent en fonction des contextes d'usage, politiques, idéologiques et sociaux. Il peut renvoyer à la 
«reconnaissance des groupes pour mettre fin à leur stigmatisation, l'autodétermination, la distribution des ressources et les droits politiques»; il peut également "prendre en compte les conditions socio-économiques et politiques de l'exercice du pouvoir, sans pour autant interroger structurellement les inégalités sociales », ou encore désigner «la capacité de conduire rationnellement son existence, de faire des choix $»^{40}$. Le corollaire est que la reconnaissance du statut de victime peut prendre des significations variées selon l'importance accordée à l'individu et aux conditions sociales de la victimation. On aurait ainsi tort de penser que les féministes des années 1970 enfermaient systématiquement les femmes dans la catégorie " victimes » : se dire victime et être reconnue comme telle devait permettre d'en sortir. Cependant, la perspective mobilisée pour qualifier le sujet de l'action change. Utiliser survivor permet de se dire survivant-e-s, et d'être dite survivant-e-s, tout en mettant en avant les capacités individuelles pour en sortir et valoriser l'expérience de la victimation. Outre des transformations endogènes à l'espace féministe, l'usage du terme survivor fait plus largement écho à son important succès dans la société étatsunienne, dans la lutte contre le cancer du sein par exemple ${ }^{41}$.

21 La figure du survivor permet également de ne pas exclure certaines victimes, ce qu'un sujet totalisant risque de faire. Contrairement à la plupart des actions qui visent à rassembler des femmes et se concentrent sur la formation d'un sujet politique "femmes ", The Clothesline Project est mixte à UCLA. Le collectif d'organisation est non seulement composé de personnes aux identités de genre différentes, mais la performance a lieu dans la cour de l'université et est ouverte à toutes et tous. Chacun-e peut venir pour témoigner des violences vécues ou de celles dont il ou elle a été le témoin. L'abandon de la non-mixité renvoie en partie aux controverses au sein de l'espace féministe. Certaines féministes, notamment celles qui ont contribué à rendre visible les violences dans l'espace social, ont été critiquées par les féministes noires et lesbiennes pour avoir homogénéisé le sujet «femmes" sans rendre compte de la multiplicité des rapports sociaux qui construisent les sujets. Au même titre qu'il n'y pas «la» femme, mais «les» femmes, la diversité des expériences, liées au genre mais aussi aux rapports sociaux de classe et race, perturbe l'idée qu'une oppression commune serait constitutive du sujet « femmes ». Souligner les catégories de violences, et non les catégories de femmes, permet de contourner ces écueils tout en s'adressant à un public relativement large. La figure du survivor répond peu à la question, si polémique au sein des féminismes, de la prise en compte des «différences entre femmes $»^{42}$ : le fait d'avoir subi des violences unit un sujet collectif et permet de subsumer les particularités socio-raciales.

Contrairement aux théories qui soutiennent que le féminisme s'est considérablement affaibli, ou encore qu'il a été transformé par le néolibéralisme en adoptant une perspective individualiste, les exemples donnés dans cet article montrent que les actions et les mobilisations féministes sont travaillées par le contexte dans lequel elles évoluent, et que les enjeux de leur lutte sont ainsi redéfinis. Souligner les reconfigurations d'un mouvement politique permet justement d'éviter une lecture binaire et normative de ses transformations. The Clothesline Project illustre la façon dont le sujet de la représentation politique est déstabilisé par la multiplication et par l'addition d'actions particulièrement structurées. Sans reposer sur une identité collective, construite et représentée par un mouvement social, on peut penser qu'un sujet politique, au sens large du terme, se dessine à travers la figure du survivor. Ce sujet 
est «trouble », il ne se fonde pas nécessairement sur la prise de conscience de l'effet de rapports sociaux clairement identifiés, mais sur le fait d'avoir subi une violence sexuelle. En mettant en avant la capacité d'agir des victimes et en essayant de rendre compte de la diversité des cas de violence, la constitution de ce sujet promeut une approche non-excluante et anti-victimaire, mais elle laisse de côté la question des causes de la violence. Pour prolonger la réflexion, on pourrait interroger les effets pratiques d'un tel changement langagier, le fait d'utiliser « victime » ne traduit en effet pas nécessairement une approche victimisante des personnes ayant subi des violences. Et l'utilisation récurrente de survivor ne signifie pas non plus que la capacité d'agir soit nécessairement mieux prise en compte dans les actions de dénonciation des violences et d'accompagnement des personnes victimes. Mobiliser "victime» ou "survivor " sous-tend nécessairement des tensions entre la valorisation des dynamiques structurelles à l'œuvre dans la victimation et celle de la capacité d'agir individuelle. A l'instar de la notion d'empowerment, il importe d'aller au delà des termes et des notions employés, de se pencher sur leurs usages sociaux et les pratiques militantes pour mieux comprendre comment évoluent les mouvements féministes.

\section{BIBLIOGRAPHIE}

Bacchetta, Paola, Jules Falquet et Norma Alarcón (eds.). « Théories féministes et queer décoloniales ». Les cahiers du Cedref 18 (2011).

Bacqué, Marie-Hélène et Carole Biewener. L'empowerment : une pratique émancipatrice. Paris : La Découverte, 2013.

Baumgardner, Jennifer et Amy Richards. Manifesta : Young Women, Feminism, and the Future. New York : Farrar, Straus \& Giroux, 2000.

Bronstein, Carolyn. Battling Pornography. The American Feminist Anti-pornography Movement, 1976-1986. Cambridge : Cambridge University Press, 2011.

Broqua, Christophe et Françoise Loux (eds.). « Sida : deuil, mémoire, nouveaux rituels ». Ethnologie française 28. 1 (janvier-mars 1998).

Brownmiller, Susan. Against our Will. Women and Rape. New York : Simon Schuster, 1975.

Campbell, Rebecca et Patricia Yancey Martin. « Services for Sexual Assault Survivors ». In Sourcebook on Violence against Women. Thousand Oaks : Sage, 2001.

Crenshaw, Kimberlé. « Cartographie des marges : intersectionnalité, politiques de l'identité et violences contre les femmes de couleur ». Cahiers du genre 39 (2005) : 51-82.

Davis, Angela. Women, Race and Class. New York : Random House, 1981.

Delage, Pauline. « Violence conjugale / Domestic violence. Sociologie comparée d'une cause féministe (France / Etats-Unis. 1970-2013 ». EHESS, 2014.

Delphy, Christine. L'ennemi principal. Paris : Syllepse, 2013. 
Dorlin, Elsa. Black feminism. Anthologie du féminisme africain-américain 1975-2000. Paris :

L'Harmattan, 2008.

Ehrenreich, Barbara. Bright-sided : How the Relentless Promotion of Positive Thinking has Undermined America. New York : Macmillan, 2009.

Fassin, Éric. « Le date rape aux États-Unis ». Enquête 5 (1997).

Ferree, Myra Marx et Patricia Yancey Martin (eds.). Feminist Organizations : Harvest of the New Women's Movement. Temple : Temple University Press, 1995.

Fraser, Nancy. « Multiculturalisme, anti-essentialisme et démocratie radicale ». Cahiers du genre 39 (2005) : 27-50.

Griffin, Susan. « Rape : The All-American Crime ». Ramparts Magazine (septembre 1971) : 26-35.

Hartsock, Nancy. The Feminist Standpoint Revisited and Other Essays. Colorado : Westview Press, 1998.

Heywood, Leslie et Jennifer Drake (eds.). Third Wave Agenda : Being Feminist, Doing Feminism. Minneapolis : University of Minnesota Press, 1997.

hooks, bell. Ain't I a Woman : Black Women and Feminism. Boston : South End Press, 1981.

Jaspard, Maryse. Les violences contre les femmes. Paris : La découverte, 2005.

Labry, Manon. « Riot Grrrls américaines et réseaux féministes « underground » français ». Multitudes 3 (2010) : 60-66.

Larsen, Mary Anne. « Grassroots Centers and National Task Forces : A Herstory of the Anti-Rape Movement ». Aegis (automne 1981) : 46-52.

Moraga, Cherríe (ed.). This Bridge Called my Back: Writings by Radical Women of Color. Watertown, MA : Persephone Press, 1981.

Naples, Nancy (ed.). Community Activism and Feminist Politics : Organizing across Race, Class, and Gender. New York : Routledge, 2012

Pollak, Michael et Nathalie Heinich. «Le témoignage ». Actes de la recherche en sciences sociales 62.1 (1986) : 3-29.

Price, Lisa Sydney. Feminist Frameworks : Building Theory on Violence against Women. Halifax :

Fernwood, 2005.

Schmitt, Frederika E. et Patricia Yancey Martin. « Unobtrusive Mobilization by an Institutionalized Rape Crisis Senter. "All We Do Comes from Victims” ». Gender \& Society 13.3 (1999) : 364-384.

Schneider, Elizabeth M. Battered Women and Feminist Lawmaking. New Haven : Yale University Press, 2008.

Staggenborg, Suzanne et Verta Taylor. "Whatever happened to the women's movement?». Mobilization. An International Quarterly (2005) : 37-52.

Taylor, Verta et Leila J. Rupp. « Women's Culture and Lesbian Feminist Activism : A Reconsideration of Cultural Feminism ». Signs (1993) : 32-61.

Tilly, Charles. « Les origines du répertoire de l'action collective contemporaine en France et en Grande-Bretagne ». Vingtieme siecle. Revue d'histoire (1984).

Thompson, Becky. « Multiracial Feminism : Recasting the Chronology of Second Wave

Feminism ». Feminist Studies (2002) : 337-360. 


\section{NOTES}

1. En français : le projet du fil à linge.

2. Staggenborg, Suzanne et Verta Taylor. "Whatever happened to the women's movement?». Mobilization. An International Quarterly (2005) : 37-52.

3. La manière de voir et comprendre le monde étant liée au positionnement social, la production de savoirs n'est pas exempte de biais socio-raciaux. Sur l'épistémologie du point de vue, voir Hartsock, Nancy. The Feminist Standpoint Revisited and Other Essays. Colorado: Westview Press, 1998.

4. Ferree, Myra Marx et Patricia Yancey Martin (eds.). Feminist Organizations: Harvest of the New Women's Movement. Temple: Temple University Press, 1995 ; Naples, Nancy (ed.). Community Activism and Feminist Politics : Organizing across Race, Class, and Gender. New York: Routledge, 2012.

5. Voir hooks, bell. Ain't I a Woman: Black Women and Feminism. Boston : South End Press, 1981 ; Moraga, Cherríe (ed.). This Bridge Called my Back : Writings by Radical Women of Color. Watertown, MA : Persephone Press, 1981. Pour des recueils de textes traduits en français, voir Dorlin, Elsa. Black feminism. Anthologie du féminisme africain-américain 1975-2000. Paris : L'Harmattan, 2008; Bacchetta, Paola, Falquet, Jules et Norma Alarcón (eds.). "Théories féministes et queer décoloniales ». Les cahiers du Cedref 18 (2011).

6. Pour illustrer la diversité des histoires et des répertoires d'action, il convient alors d'utiliser le pluriel et évoquer non pas « le » féminisme mais bien « les féminismes ».

7. Dans la mesure où la question de la qualification juridique et la nature des violences sexuelles n'est pas au cœur de cet article, on utilisera indifféremment les termes de viol ou violences sexuelles.

8. Le slogan "This is what a feminist looks like» (en français "Voici à quoi ressemble une féministe ») est utilisé comme une forme de détournement du stigmate que porte le fait d'être féministe, en réhabilitant cet «être féministe » comme un sujet politique légitime. J'utilise ici le pluriel pour insister sur la pluralité des mouvements, des groupes et des approches féministes, un positionnement qui se trouve au cœur de cet article.

9. Delage, Pauline. «Violence conjugale / Domestic violence. Sociologie comparée d'une cause féministe (France / Etats-Unis. 1970-2013 ». EHESS, 2014.

10. Albenga, Viviane, Alban Jacquemart et Laure Bereni (eds.), « Appropriations ordinaires des idées féministes ", Politix 109 (2015).

11. Weldon, S. Laurel. Protest, Policy, and the Problem of Violence Against Women: A Cross-National Comparison. Pittsburgh : University of Pittsburgh Press, 2002.

12. Bronstein, Carolyn. Battling Pornography. The American Feminist Anti-pornography Movement, 1976-1986. Cambridge: Cambridge University Press, 2011: 25-37. L'expérience de femmes militantes au sein de l'extrême gauche et de la gauche contre-culturelle a pu permettre à certaines de se rendre compte qu'aucun milieu, aussi politisé soit-il, n'est exempt des logiques sexistes existantes dans le monde social. Pour ne prendre qu'un exemple des plus emblématiques, rappelons la réponse de Stokely Carmichael lorsque des militantes l'interrogent sur la place des femmes au sein de l'organisation Student Non-violent Co-ordinating Committee : « allongées » dira-t-il.

13. Griffin, Susan. « Rape : The All-American Crime ». Ramparts Magazine (septembre 1971) : 26-35.

14. Brownmiller, Susan. Against our Will. Women and Rape. New York : Simon Schuster, 1975.

15. Voir notamment la revue Feminist Alliance Against Rape Newsletter-Aegis, http://www.faaraegis.org/, consulté le 12 octobre 2014.

16. Organisée annuellement, la première manifestation de ce type se déroule en 1977.

17. Davis, Angela. Women, Race and Class. New York : Random House, 1981.

18. Voir également Schneider, Elizabeth M. Battered Women and Feminist Lawmaking. New Haven : Yale University Press, 2008. 
19. Price, Lisa Sydney. Feminist Frameworks : Building Theory on Violence against Women. Halifax : Fernwood, 2005.

20. Schmitt, Frederika E. et Patricia Yancey Martin. «Unobtrusive Mobilization by an Institutionalized Rape Crisis Senter. "All We Do Comes from Victims" ». Gender \& Society 13.3 (1999) : 364-384 ; Campbell, Rebecca et Patricia Yancey Martin. «Services for Sexual Assault Survivors ». in Sourcebook on Violence against Women. Thousand Oaks : Sage, $2001: 227-246$.

21. Larsen, Mary Anne. «Grassroots Centers and National Task Forces : A Herstory of the AntiRape Movement ». Aegis (automne 1981) : 46-52.

22. Ferree, Myra Marx et Patricia Yancey Martin (eds.). op. cit.

23. Fassin, Éric. « Le date rape aux États-Unis ». Enquête 5 (1997).

24. Voir le site national du projet, http://www.clotheslineproject.org/, consulté le 12 octobre 2014.

25. Fassin, Éric. art. cit.

26. Comme dans l'extrait de carnet de terrain introductif, je féminise le terme à dessein puisque la mixité des membres du collectif est probablement l'une des ruptures importantes avec les modes d'action des féministes radicales.

27. Tilly, Charles. «Les origines du répertoire de l'action collective contemporaine en France et en Grande-Bretagne ». Vingtieme siecle. Revue d'histoire (1984) : 99.

28. Voir par exemple http://takebackthenight.org/ consulté le 24 avril 2015.

29. Texte original : «...on each shirt is a personal message from someone who has survived rape, abuse, incest, or hate crimes. Although some shirts are made in remembrance of those who did not survive. Some of these messages are angry, some are vulgar, some are forgiving, some are simply factual retellings. The Line gives participating survivors a way to speak out without fear ", voir http://clotheslineproject.wordpress.com/about/, consulté le 12 octobre 2014.

30. Taylor, Verta et Leila J. Rupp. «Women's Culture and Lesbian Feminist Activism: A Reconsideration of Cultural Feminism ». Signs (1993) : 32-61.

31. Labry, Manon. "Riot Grrrls américaines et réseaux féministes « underground » français ». Multitudes 3 (2010) : 60-66.

32. Fassin, Éric. art. cit.

33. Pollak, Michael et Nathalie Heinich. «Le témoignage ». Actes de la recherche en sciences sociales 62.1 (1986) : 3-29.

34. L'importance du témoignage et la forme même de The Clothesline Project rappellent l'initiative de The Aid Memorial Quilt, une autre action mise en œuvre dans les années 1980 au sein des mouvements de lutte contre le Sida et qui consiste à coudre un patchwork de témoignages et de noms pour commémorer la mort de certaines des premières victimes de l'épidémie. Il y a là probablement une forme de transfert de pratiques militantes, fondé sur une "mémoire forte " pour rendre visible la cause des violences, et rendre hommage aux victimes. Voir http:// www.aidsquilt.org/about/the-aids-memorial-quilt, consulté le 12 octobre 2014. Broqua, Christophe et Françoise Loux (eds.), «Sida : deuil, mémoire, nouveaux rituels ». Ethnologie française 28.1 (janvier-mars 1998).

35. Cette liste provient du site national. Il est cependant probable que la correspondance des couleurs et des catégories de violences diffère d'un campus à l'autre. Voir http:// www.clotheslineproject.org/aboutshirts.htm, consulté le 12 octobre 2014.

36. Texte original : « to raising awareness about sexual and gender violence issues on campus and around the world», http://clotheslineproject.wordpress.com/about/, consulté le 12 octobre 2014.

37. Texte original : «to increase awareness of the impact of violence against women, to celebrate a woman's strength to survive and to provide another avenue for her to courageously break the silence that often surrounds her experience ", http://www.clotheslineproject.org/start.htm, consulté le 12 octobre 2014. 
38. Delphy, Christine. L'ennemi principal. Paris : Syllepse, 2013.

39. Je m'appuie ici sur la distinction, faite notamment dans l'ENVEFF, entre la victimation qui consiste à reconnaître un statut de victime, et la victimisation, qui tend à enfermer dans un tel statut. Jaspard, Maryse. Les violences contre les femmes. Paris : La découverte, $2005: 19$.

40. Je reprends ici la définition des modèles radical, libéral et néo-libéral, rattachés aux sens de la notion d'empowerment, voir Bacqué, Marie-Hélène et Carole Biewener. L'empowerment: une pratique émancipatrice. Paris : La Découverte, 2013 : 15-17.

41. Ehrenreich, Barbara. Bright-side: How the Relentless Promotion of Positive Thinking has Undermined America. New York : Macmillan, 2009.

42. Crenshaw, Kimberlé. « Cartographie des marges : intersectionnalité, politiques de l'identité et violences contre les femmes de couleur ». Cahiers du genre 39 (2005) : 51-82 ; Fraser, Nancy. « Multiculturalisme, anti-essentialisme et démocratie radicale ». Cahiers du genre 39 (2005) : 27-50.

\section{RÉSUMÉS}

Phénomène tabou jusque dans les années 1970, le problème des violences sexuelles a commencé à apparaître dans l'espace social grâce à la construction d'un cadre de compréhension féministe reliant dimensions personnelles et politiques, violences privées et dynamiques de genre. Multiples, les mouvements de dénonciation des violences se sont transformés, les approches politiques et les modes d'action ayant par exemple été renouvelées dans les années 1990. A Cape Cod, des étudiantes décident de lancer une campagne de dénonciation des violences en exposant des tee-shirts censés symboliser des survivors (survivantes de violences), dans une performance intitulée The Clothesline Project, qui se diffuse ensuite dans le reste des Etats-Unis. En analysant cette action, cet article propose de penser ce que cette performance révèle des évolutions du féminisme. Si The Clothesline Project vise à faire prendre conscience des violences sexuelles, les femmes ne sont pas le sujet principal des revendications, et les racines du problème n'apparaissent pas. Cette action atteste des reconfigurations des politiques de la représentation dans le féminisme contemporain.

Taboo until the 1970s, the problem of sexual violence appeared in the social space thanks to a feminist framework, linking personal and political dimensions, private violence and gender dynamics. Characterized by their diversity, the feminist movements which denounce violence have undergone transformations; for instance, their political approaches and modes of action have been renewed since the 1990s. In Cape Cod, students launched a campaign displaying tee shirts which symbolized survivors of sexual violence. This performance, called The Clothesline Project, then spread throughout the United States. By analyzing this action, this article sets out to highlight the evolutions of feminist movements. If The Clothesline Project aims at raising awareness about the issue of violence, women are no longer the main political subject represented, and the roots of the problem are not apparent. This action shows how the politics of representation are reconfigured in contemporary feminisms. 
INDEX

Mots-clés : féminismes, violences sexuelles, action collective

Keywords : feminisms, sexual violence, collective action

\section{AUTEUR}

\section{PAULINE DELAGE}

Chercheuse FNS senior UNIL-CEG

pauline.delage@unil.ch 